\title{
18F FDG PET-CT Imaging in Tuberculosis
}

\author{
1Shamim M F Begum and ${ }^{2}$ Md Abdus Shakur Khan \\ 1National Institute of Nuclear Medicine and Allied Sciences (NINMAS), Dhaka, Bangladesh, 2National Institute of Diseases of the Chest \\ and Hospital, Dhaka, Bangladesh \\ Correspondence Address : Prof. Dr. Shamim Momtaz Ferdousì Begum, Email pragyna06@yahoo.com
}

\begin{abstract}
Tuberculosis (TB) is the second highest infective cause of death worldwide and the global impact of TB is very important. Among all the TB burden $\mathrm{WHO}$ regions, $40 \% \mathrm{~TB}$ cases accounts in the South East Asian region. It has become a medical emergency not only in developing countries but also in some high-income countries. The rising incidence of multidrug resistance (MDR) TB and HIV co-infection has increased the morbidity and mortality of TB despite the availability of cheap and effective treatment. The diagnosis of active TB is almost similar over the world. Conventional radiography and Computed Tomography $(\mathrm{CT})$ imaging play a crucial role in the diagnosis of TB. But these conventional imaging are often nonspecific and unable to provide a definitive diagnosis in cases of atypical and heterogeneous presentation. The signs of TB may mimic other diseases in conventional imaging. The introduction of new imaging tool Fluorine18 Fluorodeoxyglucose Positron Emission Tomography-Computed Tomography (18F FDG PET-CT) opens the door to evaluate its potential application in TB. The role of this new imaging tool in TB imaging has been well documented. 18F FDG PET-CT may assist in early diagnosis, facilitate differentiation between malignancies and TB, identification of extrapulmonary TB, staging of $T B$, and in assessment of treatment response. Therefore, familiarity with the spectrum of imaging features and understanding the use of 18F FDG PET-CT in diagnosis and management of TB is important, specially for referring clinicians and the reporting nuclear medicine specialists in TB burden country like Bangladesh. This article reviews the main applications, pattern of imaging spectrum with limitations of 18 F FDG PET-CT in TB.
\end{abstract}

Key words: Fluorine18 fluorodeoxyglucose, PET- CT, tuberculosis.

\section{INTRODUCTION}

Tuberculosis (TB) is the second highest infective cause of death worldwide and most common presentation of active infection (1). According to the World Health Organization (WHO) Global tuberculosis report 2015, estimated 9.6 million people have developed TB and 1.5 million people have died annually. Among all the WHO regions, $40 \%$ TB cases accounts in the South East Asian region (2). Over 95\% of TB deaths occur in low and middle income countries. This disease has become a medical emergency not only in developing countries but also in some high-income countries, because of migration of people from TB endemic area to higher-income areas, frequent co-infection with HIV/AIDS, and development of drug-resistant strains of TB (3). Hence the global impact of TB is very important. The rising incidence of multidrug resistance (MDR) TB and HIV co-infection has increased the morbidity and mortality of TB despite the availability of cheap and effective treatment. Globally, 132120 cases of multidrug-resistant $\mathrm{TB}$ or rifampicin-resistant $\mathrm{TB}$ (MDR/RR-TB) were detected and notified in 2015, and 124990 cases were enrolled on treatment (2). The immune-compromised patients are more prone to be infected with MDR-TB, and resistance strains have a greater tendency to involve extrapulmonary sites. Early diagnosis prompts effective treatment and leads to a reduced transmission of TB (4).

The diagnosis of active TB is almost similar over the world. Sputum culture is the gold standard for diagnosis. Traditional Mantoux test is also used to assess immunological response (5). Conventional radiography and Computed Tomography (CT) imaging play a crucial role in the diagnosis of TB. Plain chest radiography is the mainstay in pulmonary TB or lung parenchymal disease, while CT is more sensitive in case of lymphadenopathy and early bronchogenic change. 
But these conventional imaging are often nonspecific and unable to provide a definitive diagnosis in cases of atypical and heterogeneous presentation. The signs of TB may mimic other diseases in conventional imaging, such as neoplasms or sarcoidosis. Patients with sputum-negative pulmonary TB (PTB) and extra- pulmonary TB (EPTB) or TB with co-infection of HIV/AIDS are difficult to diagnose due to their atypical presentation and may be missed at all points of patient care. In case of drug resistance TB, the major challenge is treatment failure and treatment monitoring for therapy response is very essential (6). The introduction of new imaging tool Fluorine18 Fluorodeoxyglucose Positron Emission TomographyComputed Tomography, (18F FDG PET-CT) opens the door to evaluate its potentiality in clinical application in TB. The value of this new imaging tool in TB imaging has been well documented through several studies. Hence familiarity with the spectrum of imaging features is required to facilitate timely diagnosis and monitoring of TB treatment $(4,7)$.

Mycobacterium tuberculosis (Mtb) is an aerobic microorganism with complex pathophysiology. It may persist in host cells in variable states; ranging from latent infection to active clinical disease. The complex pathology of the organism provides multiple molecular targets for imaging the infection and targeting therapy. 18F FDG accumulates in inflammatory cells such as neutrophils, activated macrophages, and lymphocytes at the sites of inflammation or infection. TB granulomas consist of macrophages and lymphocytes, which demonstrate high levels of glucose uptake resulting in increased uptake of $18 \mathrm{~F}$ FDG on PET imaging. In the activated lymphocyte approximately 20 fold increased glucose uptake occurs over 24 hours due to increased glycolysis $(8,9,10)$. Hence any active TB infection avidly takes up $18 \mathrm{~F}$ FDG, like pulmonary TB, tuberculoma, extrapulnomary $\mathrm{TB}$ and in other $\mathrm{TB}$ related lesions $(8,11,12,13)$. Although characterization of the infection as active or not, extent of the disease always could not be assessed properly with conventional imaging. The $18 \mathrm{~F}$ FDG PET-CT has reported promising results in determining active lesion from not active (4).

PET-CT imaging is an integral part in quality management of oncology patients in developed countries and is a growing technology in developing countries. This technology is also increasingly used for evaluation of malignancy in both high and low TB prevalent countries (7). It is an emerging noninvasive imaging tool for diagnosis, staging and assessing therapy response in many infection and inflammations (9). Therefore, understanding of the potential impact of use of 18F FDG PET-CT in diagnosis and management of $\mathrm{TB}$ is important, especially for referring clinicians and the reporting nuclear medicine specialists. This article reviews the main applications, pattern of imaging spectrum with limitations of $18 \mathrm{~F}$ FDG PET-CT in TB.

\section{PULMONARY TUBERCULOSIS}

Pulmonary tuberculosis (PTB) is the most common presentation of TB. Classically, PTB can be divided into primary tuberculosis and post primary tuberculosis. Primary TB is due to first time exposure to the Mtb and post primary TB (also termed as reactivation, secondary or adulthood TB) develops under the influences of acquired immunity (14).

In primary $\mathrm{TB}$ the multilobar consolidation may be present in $25 \%$ cases. Two third cases are resolves without sequelae. Persistent radiological scar, calcifications or mass like opacities called tuberculomas are seen in $9 \%$ cases $(4,14)$. In case of children radiologically detectable parenchymal involvement is significantly lower and accounts about $90-95 \%$ cases comparing to adults (43\%). The most common abnormality in children is lymph node enlargement TB. Hence CT scan is more sensitive for children than the plain radiography $(15,16)$. FGD PET-CT may play a potential role in the evaluation of $\mathrm{TB}$ in children, but no reported data is available. In case of post primary TB, cavitation is the radiological hallmark and evident in 20 to $45 \%$ patients, which is a reliable marker of active TB (17). Bronchial spread of TB is associated with centrilobular nodule and tree in bud lesions in CT. In case of post primary TB hilar or meditational lymphadenopathy is uncommon (18). 
Depending on the FDG PET scan pattern, Soussan et al. has identified two different patterns of TB, one is lung type pattern and another lymphatic pattern. In lung type the infection is more localized to the lung parenchyma, usually with segmental consolidation with or without cavitations surrounded by micornodules. The infective lesions are mildly hypermetabolic that is mildly FDG avid. In case of lymphatic pattern there will be system involvement with intense infection. Mild FDG avid lymphadenopathy is the characteristic of lung type pattern whereas in case of lymphatic pattern the involved hilar and mediastinal lymph nodes will be more enlarged in size with intense FDG avidity $(11,19,20)$.

Tuberculomas are the result of healed primary pulmonary $\mathrm{TB}$ and less commonly present in post primary or reactivated TB. Due to increased glucose metabolism tuberculomas accumulate 18F FDG and several studies have reported increased FDG uptake with high maximal standardized uptake value (SUV $\max$ ) in tuberculoma $(7,21)$. Study conducted by Goo et al. (22) with histologically proven pulmonary TB in 10 consecutive patients showed increased FDG uptake in 9 tuberculomas out of 10 tuberculomas. The mean \pm (SD) of SUVmax was $4.2 \pm 2.2$. A series study by Sathekge et al. (7) have evaluated the diagnostic accuracy of PET-CT for differentiating benign from malignant pulmonary nodule in the patients with active lung TB by using dual time-point FDG PET-CT (60 minutes and 120 minutes projections). He also found higher SUVmax value in tuberculoma. Similar findings were reported in dual time point PET scan to assess FDG uptake in madiastinal lymph nodes in TB endemic area by Goo et al. and Kim et al. $(22,12)$. But SUVmax values were not significantly different in benign and malignant lesions. The reported data by Chen et al. (21), who included the patient with mean 18F FDG SUVmax value less than 2.5 and their findings were in agreement with Goo et al. and Kim et al. Another study by Kaneko et al. showed higher retention indices in both benign and malignant lesions without any significant difference (23).

Through the above studies it is evident that the main limitation of $18 \mathrm{~F}$ FDG PET-CT scan is the inability to distinguish tubercular from malignant lesion, especially in TB endemic areas. Several studies have investigated the role of dual time point 18F FDG imaging to evaluate pulmonary nodule as benign or malignancy but have shown no promising role.

Pleural effusion is frequently seen in TB patients and accounts in one fourth of the patients with primary pulmonary TB and in $18 \%$ cases of post primary pulmonary TB (24). The thickened pleura in TB demonstrates diffusely increased $18 \mathrm{~F}$ FDG uptake in FDG PET- CT scan, but need to differentiate from FDG avid mesothelioma (25).

\section{DIFFERENTIATION BETWEEN ACTIVE AND INACTIVE TB}

Radiological findings when persist for long time even after complete resolution of TB, questions may arise, whether the infection is active or not. High resolution CT scan is better than chest radiography in predictive active TB with a sensitivity of $96 \%$ versus $48 \%$ (26). It has been reported that 18 F FDG PET-CT scan is able to differentiate active TB from old or inactive disease. The SUVmax of active tuberculoma is significantly higher than the inactive tuberculoma. Kim et al. studied the role of dual time point PET imaging finding in 25 patients to characterize active pulmonary tuberculoma. The SUVmax value of early and delayed images and \% Delta SUVmax values were recorded and compared. The active pulmonary tuberculoma showed statistically significantly higher values of SUVmax then inactive tuberculoma. In early images the SUVmax values were in active $\mathrm{TB}=2.3 \pm 0.75$ and inactive $\mathrm{TB}=0.79 \pm 0.15$. In delayed images values were active $=2.48 \pm 0.79$ and inactive $0.79 \pm 0.13$ and in \% Delta SUV max values were active $=8.07 \pm 7.77$ and inactive $-3.83 \pm 6.59$ ) respectively. By using the cutoff value of SUVmax 
1.05 , the sensitivity and specificity were $100 \%$ and $100 \%$ respectively. Kim et. al concluded that FDG PET can differentiate active TB from old inactive disease by imaging two time points. This procedure would be valuable in the evaluation of treatment response and follow up (12). Another recent study concluded that 18F FDG PET-CT has the potential to become a tool for monitoring the treatment response in extrapulmonary TB or MDRTB (27).

\section{EXTRAPULMONARY TUBERCULOSIS}

Extrapulmonary TB (EPTB) may affect any organ. The most commonly affected sites are lymph nodes, pleura, musculoskeletal, gastrointestinal and genitourinary tract. Immnocompromised individual (AIDS, Leukemia, lymphoma, post organ transplant), diabetics, children, the elderly, person with low socioeconomic status and alcoholics are more affected by EPTB. The AIDS endemic has also increased the incidence of EPTB $(4,28)$. Patients with extrapulmonary (EPTB) are difficult to diagnose not only due to their atypical presentation but the sample collection (like tissue or fluid) from the affected site for analysis are difficult. Invasive procedures are involved for sample collection. Hence with the recent advancement of the imaging procedure the identification of the disease with correct localization and extent of the disease is a challenge $(4,6)$.

Studies reported that tubercular lesions can be detected more by $18 \mathrm{~F}-F D G$ PET compared to CT, especially in EPTB. Sathekge et al. has evaluated the potential impact of dual-phase 18F FDG PET versus routine staging in 16 patients with lymph node TB. In nine patients total 18 lymph node lesions were identified by both early and delayed images of PET scan, but on CT scan 9 lesions out of 18 were missed in five patients, four of them had only extrapulmonary lymph node involvement (7). PET-CT scan may show increased peripheral uptake of FDG with central hypometabolism, depending on the amount of caseation. All affected lymph node groups can be identified in single setting and allows selection of most suitable group of lymph node for biopsy (29).

Musculoskeletal TB accounts for about $3 \%$ of all TB and reported data showed more frequent spinal involvement (around $50 \%$ spinal involvement). Diagnosis of spinal involvement is difficult due to lack of specific morphological imaging findings. Failure in timely diagnosis and initiation of treatment leads to serious complication like vertebral collapse, spinal compression or deformity. MRI is the most sensitive modality of early diagnosis (4). Several studies showed the promising role of 18F FDG PET- CT for the diagnosis of spinal TB. In a recent study Skaf et al. reported that FDG scan is highly sensitive and image quality is not affected by metal artifact over other imaging modalities (30). Another recent review by Rivas-Garcia et al. on the image findings in Pott's disease has emphasized the role of advanced imaging such as $18 \mathrm{~F}$ FDG-PET in the assessment of extent of lesion, to guide for selection of biopsy site, to assist in surgery planning, and to contribute in follow-up evaluation (31). Gartz et al. studied on 16 patients with suspected spondylitis, and differentiate osteodegenerative changes from infective change by measuring the target to background ratio of FDG uptake. The authors concluded that 18F FDG PET-CT was superior to MRI and conventional nuclear medicine imaging (with 67Ga-citrate and 99mTc-MDP (32).

These findings highlight the potential role of FDG PET-CT in demonstrating lesion extent, in detecting additional lesions missed on morphological imaging, and in serving as a guide for biopsy with aspiration for culture for musculoskeletal TB.

Abdominal Tuberculosis is associated with abdominal lymphadenopathy. Abdominal lymph nodes are best evaluated by CT and for liver involvement MRI plays an added role. Only few reports are available on $18 \mathrm{~F}$ 
FDG PET-CT imaging in abdominal TB and showed that the imaging pattern is nonspecific and varied (33).

In case of central nervous system the MRI is superior to the other morphological imaging and 18F FDG PET-CT imaging may complement the other modalities.

\section{TB CO-INFECTION WITH HIV}

Diagnosis of pulmonary $\mathrm{TB}$ in patient who is co-infected with HIV is a challenge. The expected classical findings of pulmonary consolidation, apical involvement, cavitations are less common with presence of lymphadenopathy, pleural effusion, or a miliary pattern (34). Extrapulmonary localizations are frequent in HIV-infected patients and involve brain, pericardium, gastrointestinal tract, peritoneum and genitourinary tract (35). In absence of typical morphologic features, the functional imaging with $18 \mathrm{~F}$ FDG PET is helpful in the diagnosis TB with co infection HIV. The intensity and pattern of FDG uptake may suggest the diagnosis. Scharko et al, evaluated FDG PET findings in 15 patients infected with HIV-1. They observed distinct lymphoid tissue activation in the head and neck during the acute phase, a generalized pattern of peripheral lymph node activation at the mid-phase and involvement of abdominal lymph nodes during the late stages of HIV infection. This suggests that lymphoid tissue uptake follows a predictable sequence (36). Study by Sathekge et al. has confirmed this observation. In addition authors demonstrated that the FDG signal derived from PET imaging in patients with HIV not only correlates with viral load but is also inversely related to the CD4 cell count. Knowledge of the CD4 cell count and the viral load can therefore be used to predict whether lymph node involvement is likely due to retroviral disease or for other disorder like lymphoma or metastatic disease (37).

\section{MONITORING TREATMENT RESPONSE}

The most potential and important clinical application of $18 \mathrm{~F}$ FDG PET-CT in TB is evaluation of treatment response. The morphological change (decrease in size) of the tubercular lesion often takes longer time comparing to the molecular change. Sometimes after treatment lesions even increases in size leads to a difficult situation for the physicians and they failed to assess the response of treatment or to assess the need of modification of treatment. The 18F FDG PET-CT scan can evaluate the molecular changes related to glycolytic activity within the inflammatory lesion by measuring FDG uptake to evaluate treatment response (38) (Figure 1A-1C).
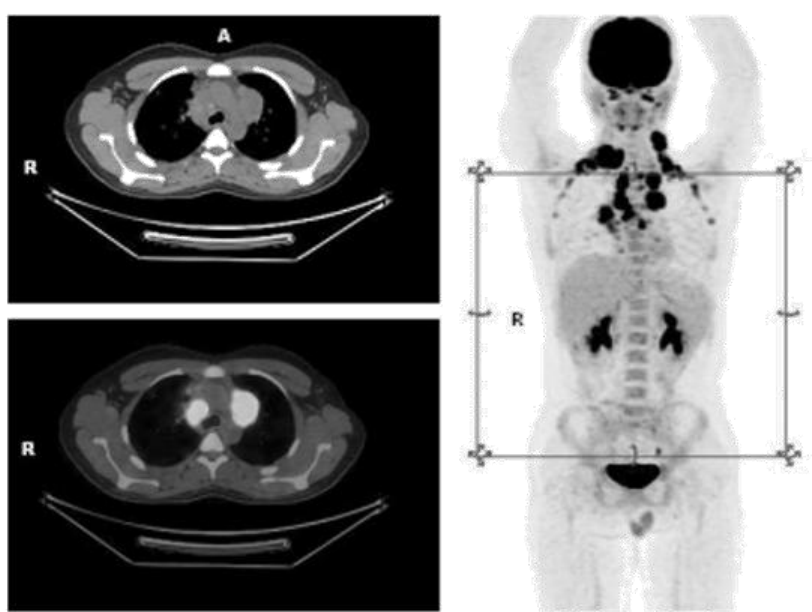

Figure 1A: A 19-year-old male with MDR tubercular lymphadeonopathy. Prior to treatment with second line of anti-TB drugs, transaxial 18F-FDG PET-CT showed multiple hypermetabolic mediastinal lymph nodes with a maximum standardized uptake value (SUVmax) of 19.8. Maximum intensity projection image revealed multiple hypermetabolic enlarged cervical, axillary and mediastinal lymph nodes.
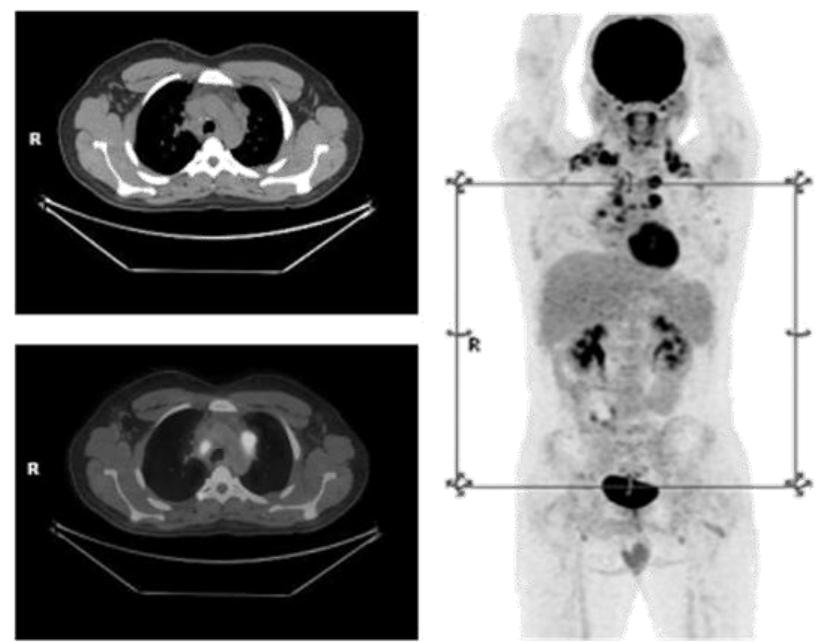

Figure 1 B: After two months treatment, PET-CT scan showed both morphologic regression and decreased metabolic activity (SUVmax) of 16.2 of mediastinal lymph nodes. 

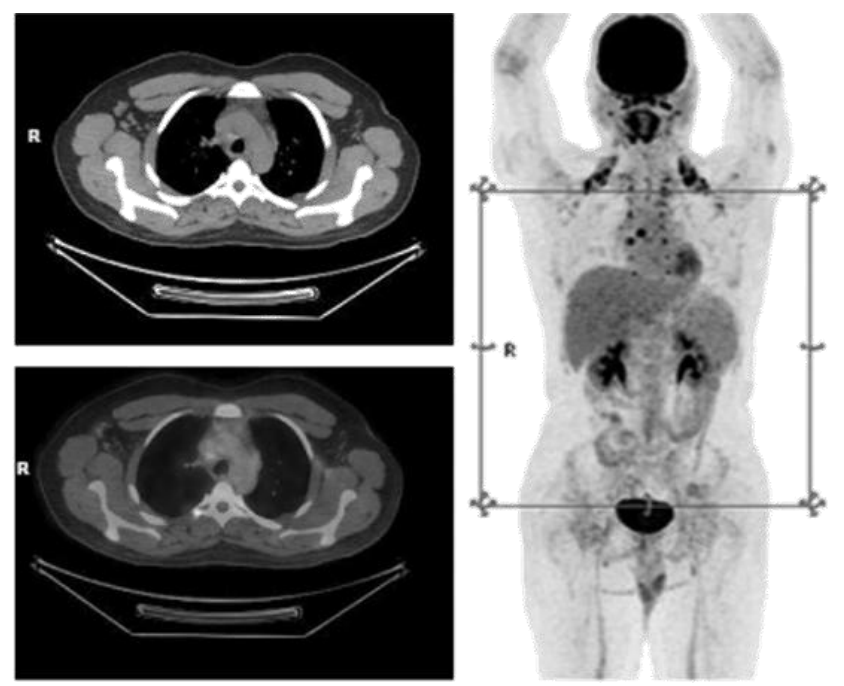

Figure1C: After eight months treatment PET-CT scan showed significant regression of metabolic activities and size of the mediastinal lymph nodes.

Martinez V et al. reported that approximately one-third reduction of SUVmax value after one month anti-TB treatment in pulmonary and extrapulmonary TB suggests good response (39). Another data has shown that SUVmax value (both early and delayed images of dual time point) of involved lymph nodes and the numbers of involved lymph node basins are significantly higher in non-responders than in responders (respective $\mathrm{P}$ values of $0.03,0.04$, and 0.002 ). Using a cutoff of 5 or more involved lymph node basins, responders could be separated from non-responders with sensitivity, specificity, positive and negative predictive value of $88 \%, 81 \%, 70 \%$, and $93 \%$, respectively. Using a cutoff value of 8.15 for the early SUVmax of lymph node basins and of 10 for the late SUVmax of lymph node basins, a comparable sensitivity of $88 \%$ with lower specificity was $73 \%$ and $67 \%$, respectively (40). The accuracy of these findings was slightly lower when FDG PET imaging was performed at 4 months after treatment initiation. Using a cutoff for SUVmax values of involved lymph nodes of 4.5 appeared more accurate. Under these circumstances, Sathekge et al. found that lymph nodes responding to TB treatment could be differentiated from nonresponsive ones with a sensitivity and specificity of $95 \%$ and $85 \%$, respectively. The number of lymph nodes with rim enhancement and low central attenuation was significantly higher in non-responders compared with responders (29).

In developing countries, multidrug-resistant and extensively drug-resistant TB has serious consequences, and monitoring of therapy response is an essential part. The treatment of MDR TB is complex and treatment duration may need to be between 9 and 18 months, and PET might provide an objective measure of treatment response and the required duration. Several studies have confirmed the value of 18 F FDG PET-CT in the follow up and evaluation of treatment response, especially in EPTB and MDR TB $(29,33,39,41,42)$.

\section{CONCLUSION}

Though 18F FDG PET-CT is not specific for TB, it plays a potential role in the evaluation of known or suspected TB, identification of EPTB with disease extension, guide biopsy from active site, staging of TB, and assessment of treatment response.

\section{REFERENCES}

1. Raviglione M, Hafner R, von Reyn CF. Tuberculosis. N Engl J Med $2013 ; 368(8): 745-55$.

2. World Health Organization (2015) Global tuberculosis report 2015 (20th edition): www.who.int/tb/publications/global_report. Assessed 23 Nov 2015.

3. Ginsberg A M, Spigelman M. Challenges in tuberculosis drug research and development. Nature Medicine 2007; 13:290-94.

4. Skoura E, Zumla A, Bomanji J. Imaging in tuberculosis. International Journal of infectious Diseases 2015; 32:87-93.

5. Mitchison DA. The diagnosis and therapy of tuberculosis during the past 100 years. Am J Respir Crit Care Med 2005; 171(7):699-706. doi: $10.1164 / \mathrm{rccm} .200411-16030 \mathrm{OE}$.

6. Maclean KA, Becker AK, Chang SD, Harris AC. Extrapulmonary tuberculosis: imaging features beyond the chest. Can Assoc Radiol J $2013 ; 64: 319-24$

7. Sathekge MM, Maes A, Pottel H, Stoltz A, van de Wiele C. Dual time-point FDG PET-CT for differentiating benign from malignant solitary pulmonary nodules in a TB endemic area. S Afr Med J 2010; $100(9): 598-601$.

8. Alfred O. Ankrah, Tjip S. van der Werf, Erik F. J. de Vries, Rudi A. J. O. Dierckx, Mike M. Sathekge, and Andor W. J. M. Glaudemans. PET/CT imaging of Mycobacterium tuberculosis infection. Clin Transl Imaging 2016; 4: 131-44. 